\title{
The Growth of Richtmyer-Meshkov Instability under Multiple Impingements
}

\author{
Tao Wang1,2, Jingsong Bai ${ }^{*}$, Ping Li¹, Bing Wang', Lei Du¹, Gang Tao², Jiaxin Xiao \\ ${ }^{1}$ Institute of Fluid Physics, China Academy of Engineering Physics, Mianyang, China \\ ${ }^{2}$ School of Energy and Power Engineering, Nanjing University of Science and Technology, Nanjing, China \\ Email: "bjsong@foxmail.com
}

Received 13 February 2016; accepted 24 April 2016; published 27 April 2016

Copyright $@ 2016$ by authors and Scientific Research Publishing Inc.

This work is licensed under the Creative Commons Attribution International License (CC BY).

http://creativecommons.org/licenses/by/4.0/

(c) (i) Open Access

\begin{abstract}
The growth of multi-mode Richtmyer-Meshkov instability under multiple impingements and the effect of initial shock strength on the growth of RMI are numerically investigated. We obtain the time evolution of turbulent mixing zone width for initial shock with different strength. The results show that the turbulent mixing zone width grows in a different manner at different stage but strictly in a similar way for the initial shock with different strength. Also, the initial shock strength has a significant effect on the growth rate of turbulent mixing zone width, especially before reshock, but can not change the growth laws in the whole process.
\end{abstract}

\section{Keywords}

Multi-Mode, Richtmyer-Meshkov Instability, Multiple Impingements, Turbulent Mixing Zone Width, Shock Strength

\section{Introduction}

As a shock wave passes through a pre-corrugated interface between two flows with different density, the Richtmyer-Meshkov instability (RMI) [1] [2] occurs due to the baroclinic vorticity deposition $\left(\nabla \rho \times \nabla p / \rho^{2}\right)$ at the interface, and induces the turbulent mixing at the late times. This phenomenon exists in a variety of man-made applications and natural phenomena such as the inertial confinement fusion (ICF) [3], deflagration-to-detonation transition (DDT) [4], high-speed combustion [5] and astrophysics (i.e. supernova explosions) [6]. It is so important that many scientists devote themselves to the study of the Richtmyer-Meshkov instability.

The impulsive models for the single-mode RMI were developed by replacing the constant acceleration of the

"Corresponding author.

How to cite this paper: Wang, T., Bai, J.S., Li, P., Wang, B., Du, L., Tao, G. and Xiao, J.X. (2016) The Growth of RichtmyerMeshkov Instability under Multiple Impingements. World Journal of Mechanics, 6, 150-158.

http://dx.doi.org/10.4236/wjm.2016.64012 
RTI (Rayleigh-Taylor instability) with an impulsive one to represent the shock wave. These models only predict the linear growth of perturbation at the early stages. The first impulsive model was proposed by Richtmyer [1] as $\dot{a}=k \Delta u A a_{0}$, where $k$ is the wave number, $\Delta u$ is the velocity jump of the interface due to the shock, $A$ is the Atwood number, $a_{0}$ is initial amplitude of perturbation. Then he found well agreement between the numerical results of light/heavy perfect gas interface and the impulsive model with replacement of the pre-shock values $A$ and $a_{0}$ by the post-shock ones $A^{\prime}$ and $a_{0}^{\prime}$. The Richtmyer model was also modified by using the average of pre- and post-shock values of $A$ and $a_{0}$ [7] [8]. Fraley [9] analytically solved the linearized perturbation equations and obtained the asymptotic linear growth, in which the inherent compressibility of gases was considered, and was first recognized by Mikaelian [10]. The linear models are based on the linear theory, so they are valid only as long as the perturbation remains small, i.e. $a(t) \ll \lambda$. To extend the range of the single-mode RMI theory to nonlinear stage, the nonlinear models were developed based on the asymptotic expansion of the linear perturbation equations. Zhang \& Sohn [11] developed a nonlinear model for the growth of a two- and three-dimensional RMI from early to late times, by treating the nonlinear problem with higher-order expansion and extending the range of validity of the series using Padé approximants. Sadot et al. [12] proposed an empirical model based on fits to experimental data and on asymptotic growth law, which is valid over Ma = $1.3-3.5$, as well as other nonlinear models such as vortex models [13], potential flow models [14] [15], etc. About the nonlinear multi-mode perturbations, a number of studies showed that the overall width of turbulent mixing zone (TMZ) evolved according to a power law $\sim t^{\theta}$ where the values ranging from $\theta=0.25$ to 1 have been proposed. Assuming no dependence on the initial conditions and neglecting the molecular dissipation effects, Mikealian [16] developed a simple model by replacing the acceleration for RTI growth rate with an impulsive acceleration as $\dot{a}=0.28 \Delta u A$ following any shock or reshock, which was also validated by some investigations [17]-[19]. This model was also extended to $t^{*}$, for which it takes $a \sim t$ for $t \leq t^{*}$ and $a \sim t^{\theta}$ for $t \geq t^{*}$ [20] [21]. All in all, these theoretical models are developed under some assumptions and limitations, and only give an approximate predict of the RMI growth. And the more studies are carried out by experiments and numerical simulations.

Hill et al. [22] studied the TMZ growth and the statistical behavior of multi-mode RMI under reshock by implementing a structure-based subgrid-scale model into the large-eddy simulation. Grinstein et al. [23] studied the effects of multi-mode initial conditions (ICs) perturbation on the mixing transition and late-time TMZ growth by using implicit large-eddy simulation. They found the significant further effects of ICs occur after reshock, and the late-time TMZ growth is higher for the long IC perturbation case than short one. Vandenboomgaerde et al. [24] investigated the nonlinear evolution of the two-dimensional single-mode RMI through experiments and simulations to explore the effect of a high initial amplitude on the growth of the perturbation, which showed the short-wavelength and long- wavelength perturbations have a different effect on the growth of the perturbation. Leinov et al. [18] systematically studied the growth of the RMI-induced turbulent mixing following a reshock by means of experiments and simulations, and found that the evolution of TMZ following the reshock is independent of its amplitude at the time of reshock but directly dependent on the strength of the reshock and grows with time in a linear manner. Thornber et al. [25] [26] investigated the influence of different three-dimensional multi-mode initial conditions on the growth of TMZ through a series of well-controlled numerical experiments without reshock. Luo et al. [27] [28] investigated the RMI of gas bubble and gas cylinder in a cylindrical converging shock tube and gave the evolving law of structural scale of the interface. Our group [29] [30] investigated the RMI with double mode perturbed interface in nonuniform flows and the effects of nonuniformity of flows on the RMI with reshock. It can be seen that the most of studies only focus on the growth of RMI excited by a single shock or reshock. And the growth of RMI under multiple impingements is rarely reported in the published literatures, which is very important in engineering applications. For example, in ICF, the initial imploding shock induces the RMI mixing of ablative shell material with the DT fuel, and the transmitted shock rebound between the shell and the center, which can affect the final compression, and thus the ability to produce energy. In this paper, the growth laws of multi-mode RMI under multiple impingements in light/heavy configuration are investigated by using an in-house parallel large-eddy simulation (LES) code MVFT.

\section{Numerical Method}

The governing equations of LES are the Favre-filtered compressible multi-viscous-flow Navier-Stokes (NS) equations which can be written as: 


$$
\left\{\begin{array}{l}
\frac{\partial \bar{\rho}}{\partial t}+\frac{\partial \bar{\rho} \tilde{u}_{j}}{\partial x_{j}}=0 \\
\frac{\partial \bar{\rho} \tilde{u}_{i}}{\partial t}+\frac{\partial\left(\bar{\rho} \tilde{u}_{i} \tilde{u}_{j}+\bar{p} \delta_{i j}\right)}{\partial x_{j}}=\frac{\partial \sigma_{i j}}{\partial x_{j}}-\frac{\partial \tau_{i j}}{\partial x_{j}} \\
\frac{\partial \bar{\rho} \bar{E}}{\partial t}+\frac{\partial\left(\bar{\rho} \tilde{u}_{j} \bar{E}+\bar{p} \tilde{u}_{j}\right)}{\partial x_{j}}=\frac{\partial\left(\sigma_{i j}-\tau_{i j}\right) \tilde{u}_{i}}{\partial x_{j}}-\frac{\partial\left(q_{j}^{l}+Q_{j}^{T}\right)}{\partial x_{j}} \\
\frac{\partial \tilde{Y}^{(s)}}{\partial t}+\tilde{u}_{j} \frac{\partial \tilde{Y}^{(s)}}{\partial x_{j}}=\frac{\partial}{\partial x_{j}}\left(\tilde{D} \frac{\partial \tilde{Y}^{(s)}}{\partial x_{j}}\right)-\frac{\partial Q_{j}^{Y}}{\partial x_{j}} \quad s=1,2, \cdots, N-1
\end{array}\right.
$$

$\bar{\rho}, \tilde{u}_{k}(k=i, j), \bar{p}, \bar{E}$ are the resolved-scale density, velocity, pressure, and total energy per unit mass, respectively; $N$ is the total number of species; $\tilde{Y}^{(s)}$ is the volume fraction of the sth fluid which satisfies $\sum_{1}^{N} \tilde{Y}^{(s)}=1 ; \tilde{D}$ is the diffusion coefficient set to $\tilde{D}=v / S_{c}$, where $v$ is the kinematic viscosity of the fluid, and $S c$ is the Schmidt number. $\sigma_{i j}=\mu_{\text {lam }}\left[\partial \tilde{u}_{i} / \partial x_{j}+\partial \tilde{u}_{j} / \partial x_{i}-2 / 3 \delta_{i j}\left(\partial \tilde{u}_{k} / \partial x_{k}\right)\right]$ is the deviatoric Newtonian stress tensor, $\mu_{\text {lam }}$ is the dynamic viscosity. $q_{j}^{l}=-\lambda_{\text {lam }} \partial \tilde{T} / \partial x_{j}$ is the resolved heat transport flux per unit time and space, where $\lambda_{\text {lam }}=\mu_{\text {lam }} c_{p} / P r_{\text {lam }}$ is the resolved heat conduction coefficient with $c_{p}$ the constant pressure specific heat and $\operatorname{Pr}_{\text {lam }}$ the Prandt number, and $\tilde{T}$ is the fluid temperature. $\tau_{i j}, Q_{j}^{T}, Q_{j}^{Y}$ are the subgrid-scale (SGS) stress tensors, heat and scalar transport fluxes, respectively, which are calculated by the Vreman SGS model [31]. The ideal gas equation of state (EOS) is adopted as the fluid EOS.

An operator splitting technique is used to decompose the physical problems, as described by Equation (1), into three sub-processes, i.e. the calculation of inviscid flux, viscous flux and heat flux. For the inviscid flux, the three-dimensional problem is simplified into three one-dimensional problems by the dimension splitting technique, and then the one-dimensional problem in each direction is solved by the two-step Lagrange-Remapping algorithm. Also, one time step is divided into four sub-steps: (i) the piecewise parabolic interpolating of physical quantities; (ii) solving the Riemann problems approximately; (iii) marching of the Lagrange equations; (iv) remapping the physical quantities back to the stationary Euler meshes. The viscous flux and heat flux are calculated based on the computed inviscid flux by using second-order spatial center difference and two-step RungeKutta time marching. For details of the numerical algorithm, the readers are suggested to Refs. [32] [33].

\section{Results and Discussions}

In the investigation, the RMI shock tube experiment conducted by Erez et al. [34] is chosen as the benchmark model for simulation, the simplified schematic and computational model is shown in Figure 1. The shock tube has a test section with a square cross section of size $80 \mathrm{~mm} \times 80 \mathrm{~mm}$. The initial multi-mode perturbed interface is located at a distance of $180 \mathrm{~mm}$ from the end wall of shock tube,

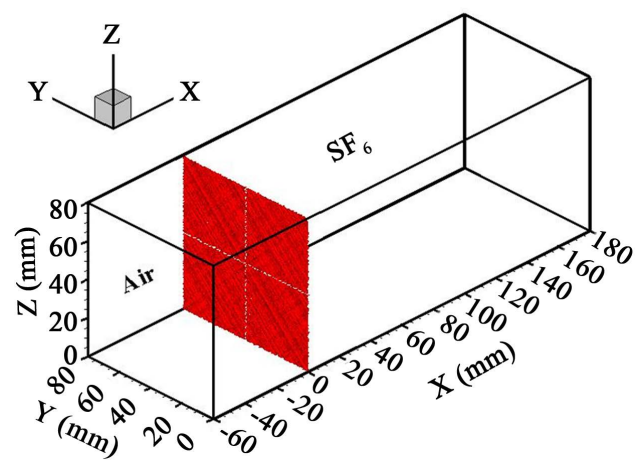

Figure 1. Simplified schematic of shock tube and computational model. 


$$
\eta(y, z)=\xi \eta_{0} \sum \cos \left(\frac{2 \pi}{\lambda_{y}}\left(y+y_{i}\right)\right) \cos \left(\frac{2 \pi}{\lambda_{z}}\left(z+z_{i}\right)\right)+\psi(y, z)
$$

where $\eta_{0}=0.14 \mathrm{~mm}$ is the amplitude of dominant modes, $\xi$ is the normalization parameter, $\lambda_{y}=\lambda_{z}$ is the wavelength in transverse directions, $y_{i}$ and $z_{i}$ are the initial phase of different modes, $\psi$ is a very small superimposed random perturbation to breaks the symmetry and accelerate the development of nonlinearity. In this simulation, the initial multi-mode perturbation is composed of eight wavelengths of $0.8,1.0,1.25,1.6,2.0,2.5,3.2,4.0 \mathrm{~mm}$. An incident shock wave with Mach number 1.2 passes through the air/SF $\mathrm{S}_{6}$ interface (Atwood number $A=0.67$ ). The initial properties of air and $\mathrm{SF}_{6}$ at $294 \mathrm{~K}$ and $1 \mathrm{~atm}$ are listed in Table 1 . The computational domain is discreted with grid resolutions $768 \times 256^{2}$ in $(x, y, z)$ directions in the parallel calculation.

When an incident shock wave impacts an initial interface in light/heavy configuration, it bifurcates into a reflected shock wave and a transmitted shock wave with decreased intensity. In a shock tube, the transmitted shock in heavy gas region travels towards the end-wall and then reflects back to the interface. The reflected shock impacts the interface again (called reshock) to generate a reflected rarefaction wave in heavy gas region. The reflected rarefaction wave will also reflect off the end-wall and re-interact with the interface to generate a reflected compression wave. The compression wave, after reflecting off the end-wall, is refracted at the interface to generate another reflected rarefaction wave in heavy gas region. The reflected rarefaction wave and compression wave are generated alternately and reverberate between the end-wall and the interface with decreasing intensity, resulting in a process of multiple impingements. These wave-interface interactions continuously deposit energy onto the turbulent mixing zone (TMZ) over the course of their passages from head to tail and advance the evolution of TMZ. Figure 2 shows the approximate one-dimensional wave diagram for the light/heavy interface configuration, which reveals the interactions of wave and interface. Figure 3 displays the time history of TMZ width, the circle symbols denote the experimental widths, the black line denotes the numerical calculated width, and the red line denotes the fitting results of numerical width before and after reshock, which is based on the least square theory. We can see that they have achieved a good agreement. The following parts will represent the growth laws of the multi-mode RMI under multiple impingements in light/heavy configuration.

Figure 4 shows the time history of TMZ width under multiple impingements for initial Mach number 1.2. It can be seen that the TMZ width grows in a different manner at different stage. After the initial shock, the perturbation starts to grow, the TMZ width evolves as a power law $\sim t^{\theta}$ where the value of $\theta$ is determined to be 0.352. After the reshock, more energy is deposited onto the interface to advance the development of TMZ, and the TMZ width evolves in time as a negative exponential law $\sim-\mathrm{e}^{-t / t^{*}}$ where the value of $t_{1}^{*}$ is 0.519 and has a difference with the Mikaelian model [16] which gives a linear growth velocity of $19.98 \mathrm{~m} / \mathrm{s}$ (blue line in figure), also with the extended model [21] [22]. Then after the following interaction of the reflected rarefaction wave with the interface, the TMZ also evolves as a negative exponential law but with a different factor $t_{2}^{*}=0.875$. Under the subsequent impingements with lower and lower intensity which have hardly influenced the evolution of TMZ, and the TMZ width, after a slight reduction caused by the reflected compression wave, evolves in an approximate linear fashion with a velocity of $2.05 \mathrm{~m} / \mathrm{s}$. The initial shock-interface interaction, as well as the subsequent reshock, reflected rarefaction and compression waves are all inherently compressible effects and form the primary mechanisms for the baroclinic vorticity deposition at the interface, each impingement advancing a faster development of the turbulent mixing zone.

So, whether the TMZ width grows in a similar way for the initial shock with different strength. We also investigate the effect of strength of initial shock on the growth of RMI, for which the Mach numbers are 1.5, 1.8, 2.0 and 2.2. Figures 5-8 show the time history of TMZ width under multiple impingements for different initial Mach number respectively, the red line denotes the fitting results of numerical width, and the blue line is the prediction of Mikaelian model [16]. It can be seen that the TMZ width grows strictly in a similar way for initial

Table 1. Initial properties of air and $\mathrm{SF}_{6}$ at $1 \mathrm{~atm}$ and $294 \mathrm{~K}$.

\begin{tabular}{cccccc}
\hline Gas & $\rho\left(\mathrm{kg} / \mathrm{m}^{3}\right)$ & $\mu_{\text {lam }}\left(10^{-5} \mathrm{~Pa} \cdot \mathrm{s}\right)$ & $\gamma$ & $\operatorname{Pr}$ & $\tilde{D}\left(10^{-5} \mathrm{~m}^{2} / \mathrm{s}\right)$ \\
\hline $\mathrm{SF}_{6}$ & 5.97 & 1.4746 & 1.09 & 0.9 & 0.97 \\
Air & 1.18 & 1.8526 & 1.40 & 0.71 & 2.04 \\
\hline
\end{tabular}




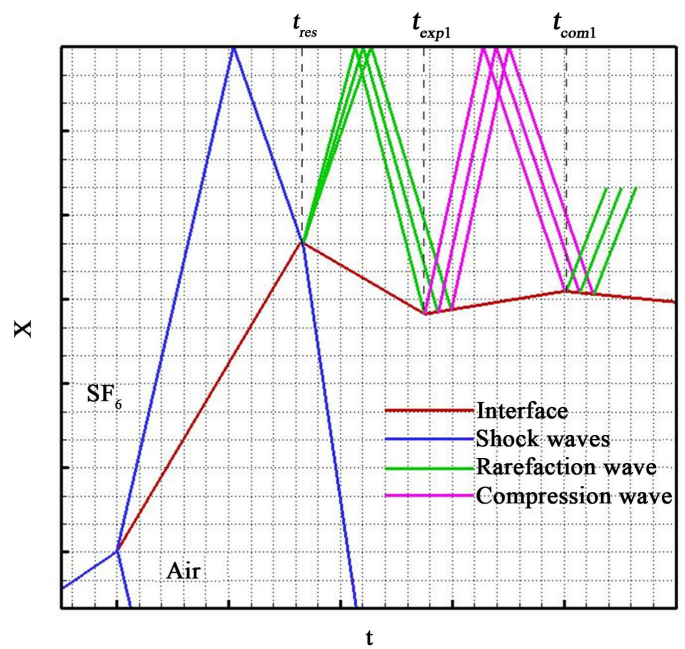

Figure 2. Approximate one-dimensional wave diagram for light/heavy configuration.

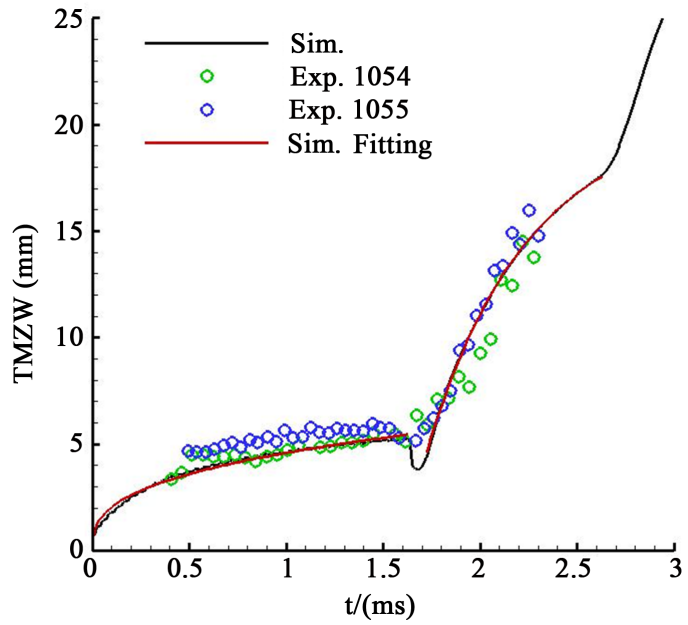

Figure 3. Time history of TMZ width.

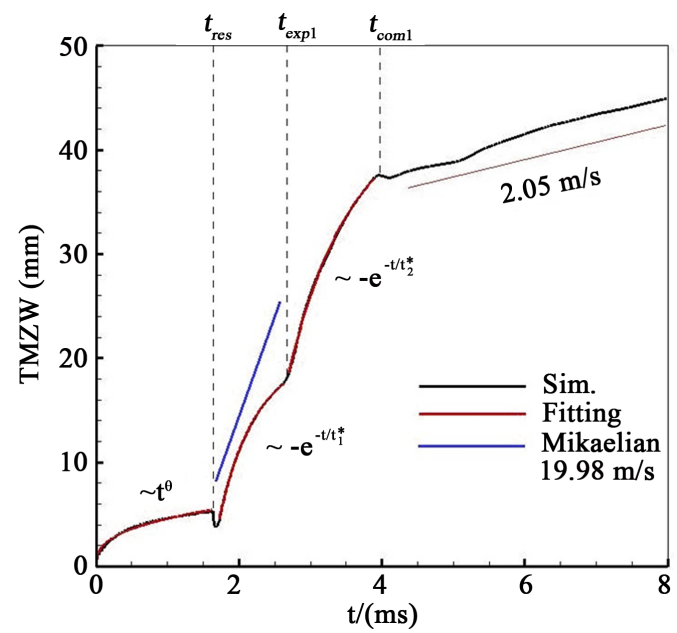

Figure 4. Time history of TMZ width under multiple impingements for initial Mach number 1.2. 


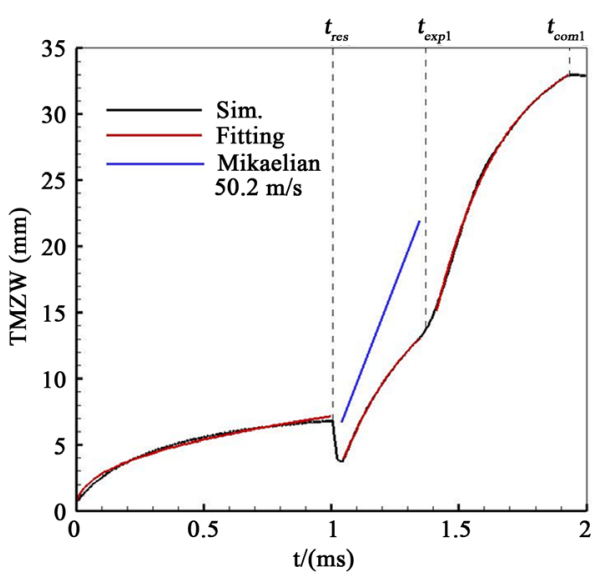

(a)

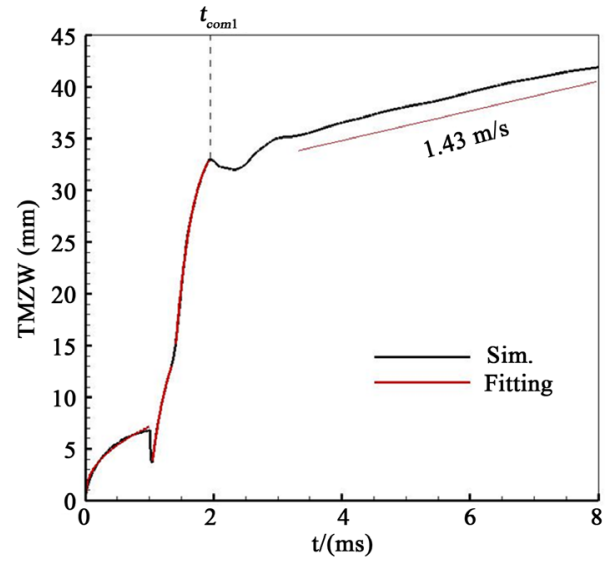

(b)

Figure 5. Time history of TMZ width under multiple impingements for initial Mach number 1.5. (a) Before the first reflected compression wave; (b) The whole process.

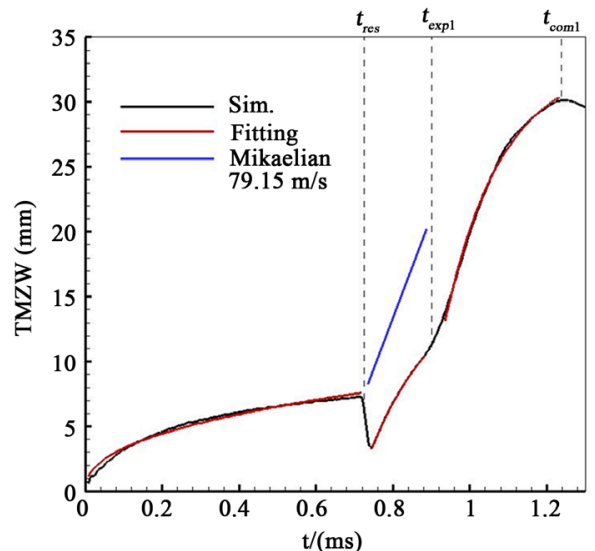

(a)

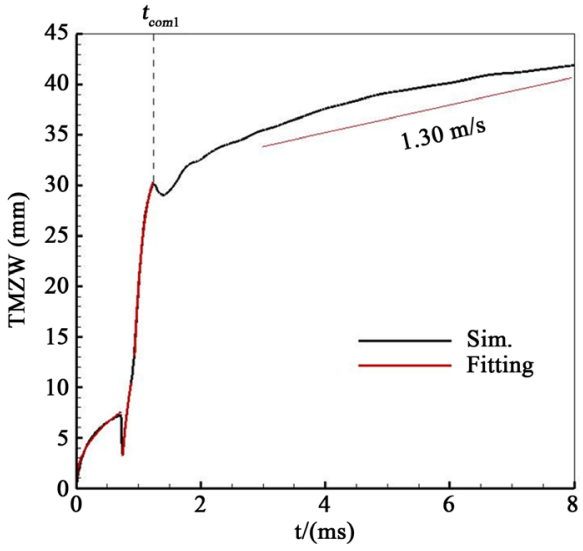

(b)

Figure 6. Time history of TMZ width under multiple impingements for initial Mach number 1.8. (a) Before the first reflected compression wave; (b) The whole process.

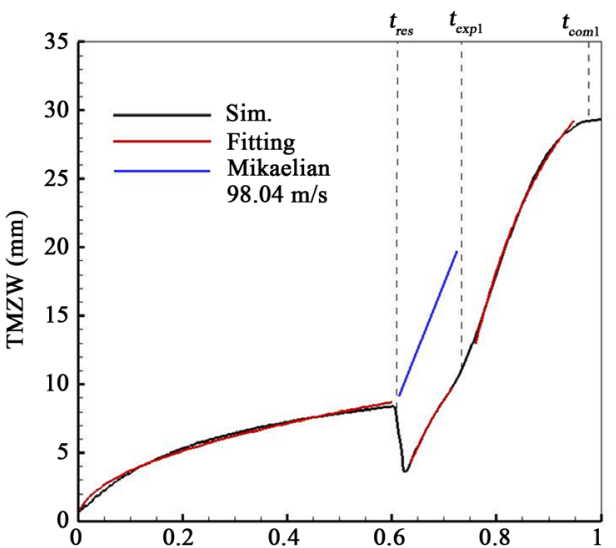

(a)

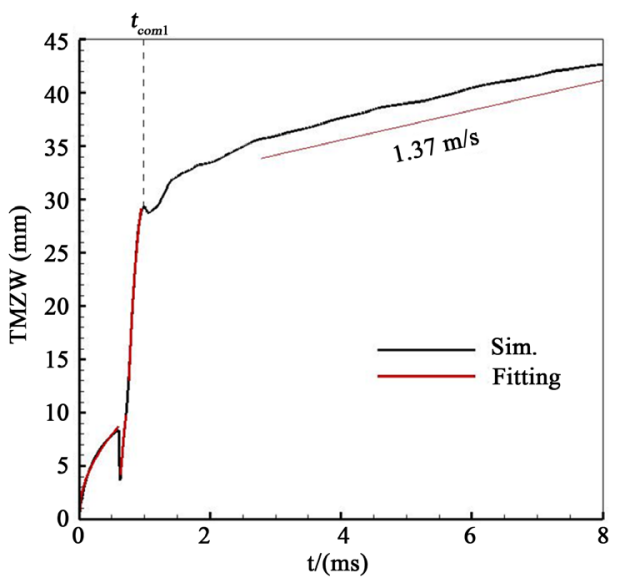

(b)

Figure 7. Time history of TMZ width under multiple impingements for initial Mach number 2.0. (a) Before the first reflected compression wave; (b) The whole process. 
shock with different strength. After the initial shock, the TMZ width evolves as a power law $\sim t^{\theta}$. After the reshock, the TMZ width evolves in time as a negative exponential law $\sim \mathrm{e}^{-t / t^{*}}$. Then after the following interacttion of the reflected rarefaction wave with the TMZ, it also evolves as a negative exponential law but with a different factor from just after reshock. Under the subsequent impingements with lower and lower intensity, the TMZ width, after a slight reduction caused by the first reflected compression wave, evolves in an approximate linear fashion.

According to the vorticity evolution equation, the stronger shock can cause the production of baroclinic vorticity with higher strength, and the perturbed interface can develop faster. Figure 9 compares the growth of TMZ width under multiple impingements for different initial Mach number, zero time is shifted to the time of arrival of reshock. It can be seen that the TMZ width surely grows faster when the initial shock is stronger. For the weaker different initial shock, there is a larger difference among the growth rate of TMZ width in the whole process but in a similar way. When the initial shock strength increases the growth of TMZ width tends to be consistency behind reshock. That is to say, the effect of initial shock mainly plays a key role in the growth of TMZ width before reshock. Table 2 lists the growth factors of TMZ width in different stage for the different initial shock Mach number. The growth factors in the corresponding stage tend to be identical along with the augment of initial shock strength.

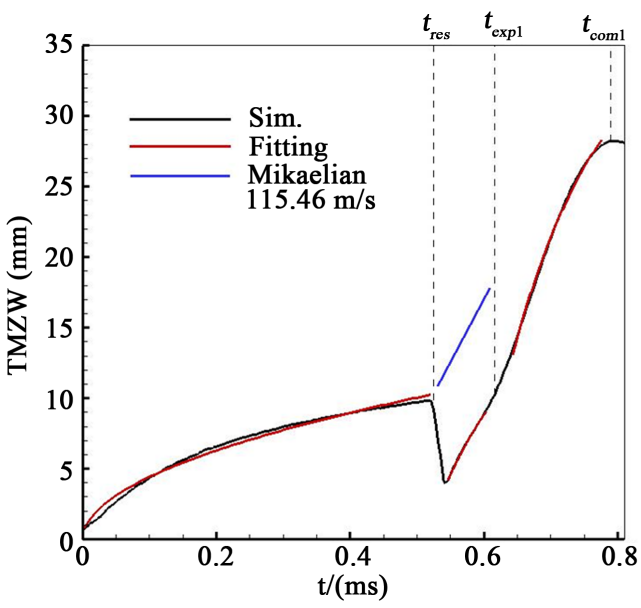

(a)

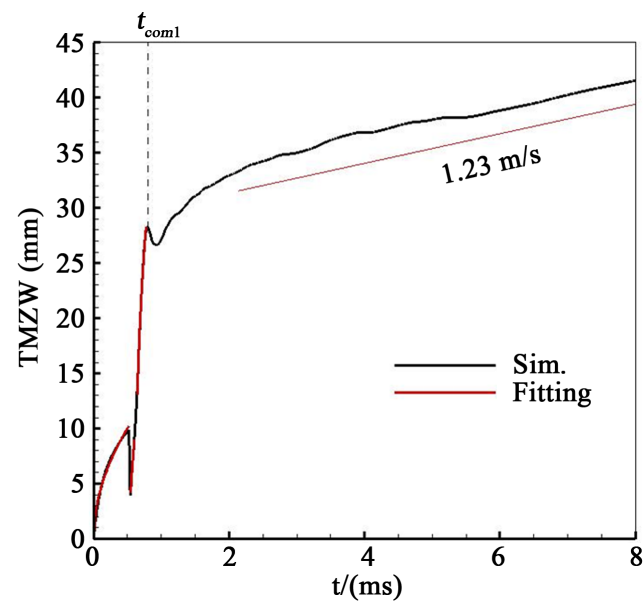

(b)

Figure 8. Time history of TMZ width under multiple impingements for initial Mach number 2.2. (a) Before the first reflected compression wave; (b) The whole process.

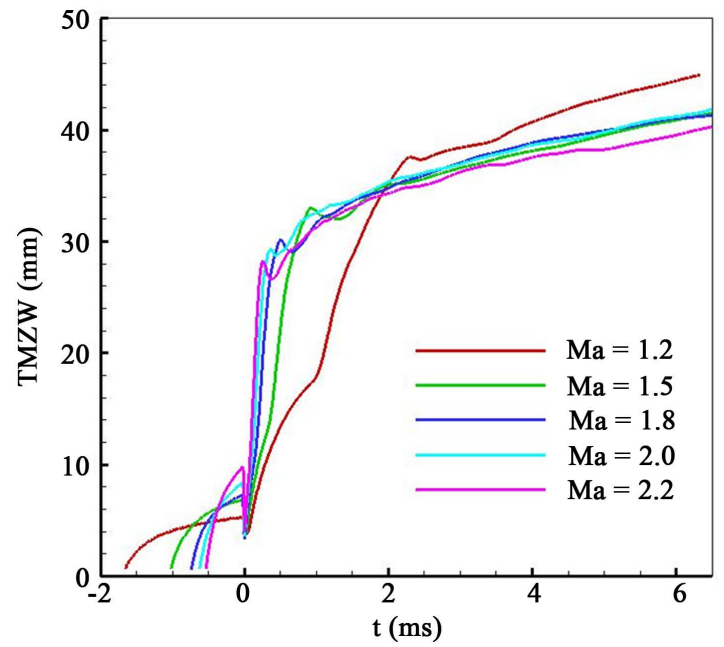

Figure 9. Time history of TMZ width under multiple impingements for different initial Mach number. 
Table 2. Growth factors of TMZ width in different stage for different initial shock Mach number.

\begin{tabular}{rccccc}
\hline Ma & 1.2 & 1.5 & 1.8 & 2.0 & $\mathbf{2}$ \\
\hline power law $\theta$ & 0.352 & 0.411 & 0.420 & 0.481 & $\mathbf{0 . 5 1 0}$ \\
Exp. law 1 $t_{1}^{*}$ & 0.519 & 0.304 & 0.185 & 0.139 & $\mathbf{0 . 1 3 4}$ \\
Exp. law 2 $t_{2}^{*}$ & 0.875 & 0.268 & 0.147 & 0.142 & $\mathbf{0 . 1 4 1}$ \\
linear law (m/s) & $\mathbf{2 . 0 5}$ & $\mathbf{1 . 4 3}$ & $\mathbf{1 . 3 0}$ & $\mathbf{1 . 3 7}$ & $\mathbf{1 . 2 3}$ \\
\hline
\end{tabular}

\section{Conclusion}

In summary, we have numerically investigated the growth of multi-mode RMI under multiple impingements and the effect of initial shock strength on the growth of RMI. The TMZ width grows in a different manner at different stage and strictly in a similar way for initial shock with different strength. After the initial shock, the TMZ width evolves as a power law. After the reshock, the TMZ width evolves in time as a negative exponential law. And after the following interaction of the reflected rarefaction wave, it also evolves as a negative exponential law but with a different factor from just after reshock. Since then the TMZ width evolves in an approximate linear fashion under the subsequent impingements with lower and lower intensity. The initial shock strength has a significant effect on the growth rate of TMZ width, especially before reshock, but can not change the growth laws in the whole process.

\section{Supports}

The authors thank supports from the National Natural Science Foundation of China (Grants No. 11372294 and 11532012), and the Foundation of National Key Laboratory of Shock Wave and Detonation Physics under Grants No. 9140C670301150C67290.

\section{References}

[1] Richtmyer, R.D. (1960) Taylor Instability in Shock Acceleration of Compressible Fluids. Communications on Pure and Applied Mathematics, 13, 297-319. http://dx.doi.org/10.1002/cpa.3160130207

[2] Meshkov, E.E. (1969) Instability of the Interface of Two Gases Accelerated by a Shock Wave. Soviet Fluid Dynamics, 4, 101-104. http://dx.doi.org/10.1007/BF01015969

[3] Amendt, P., Colvin, J.D., Tipton, R.E., et al. (2002) Indirect-Drive Noncryogenic Double-Shell Ignition Targets for the National Ignition Facility: Design and Analysis. Physics of Plasmas, 9, 2221-2233. http://dx.doi.org/10.1063/1.1459451

[4] Khokhlov, A.M., Oran, E.S. and Thomas, G.O. (1999) Numerical Simulation of Deflagration-to-Detonation Transition: The Role of Shock-Flame Interaction in Turbulence Flames. Combustion and Flame, 117, 323-339. http://dx.doi.org/10.1016/S0010-2180(98)00076-5

[5] Yang, J., Kubota, T. and Zukoshki, E.E. (1993) Application of Shock-Induced Mixing to Supersonic Combustion. AIAA Journal, 31, 854-862. http://dx.doi.org/10.2514/3.11696

[6] Arnett, D. (2000) The Role of Mixing in Astrophysics. The Astrophysical Journal Supplement Series, 127, $213-217$. http://dx.doi.org/10.1086/313364

[7] Meyer, K.A. and Blewett, P.J. (1972) Numerical Investigation of the Stability of a Shock Accelerated Interface between Two Fluids. Physics of Fluids, 15, 753-759. http://dx.doi.org/10.1063/1.1693980

[8] Vandenboomgaerde, M., Mügler, C. and Gauthier, S. (1998) Impulsive Model for the Richtmyer-Meshkov Instability. Physical Review E, 58, 1874-1882. http://dx.doi.org/10.1103/PhysRevE.58.1874

[9] Fraley, G. (1986) Rayleigh-Taylor Stability for a Normal Shock Wave-Density Discontinuity Interaction. Physics of Fluids, 29, 376-386. http://dx.doi.org/10.1063/1.865722

[10] Mikaelian, K.O. (1994) Freeze-Out and the Effect of Compressibility in the Richtmyer-Meshkov Instability. Physics of Fluids, 6, 356-368. http://dx.doi.org/10.1063/1.868091

[11] Zhang, Q. and Sohn, S. (1997) Nonlinear Theory of Unstable Fluid Mixing Driven by Shock Wave. Physics of Fluids, 9, 1106-1124. http://dx.doi.org/10.1063/1.869202 
[12] Sadot, O., Erez, L., Alon, U., Oron, D., Levin, L.A., Erez, G., Ben-Dor, G. and Shvarts, D. (1998) Study of Nonlinear Evolution of Single-Mode and Two-Bubble Interaction under Richtmyer-Meshkov Instability. Physical Review Letters, 80, 1654-1657. http://dx.doi.org/10.1103/PhysRevLett.80.1654

[13] Jacobs, J.W. and Sheeley, J.M. (1996) Experimental Study of Incompressible Richtmyer-Meshkov Instability. Physics of Fluids, 8, 405-415. http://dx.doi.org/10.1063/1.868794

[14] Layzer, D. (1955) On the Instability of Superposed Fluids in a Gravitational Field. Astrophysical Journal, 122, 1-12.

[15] Goncharov, V.N. (2002) Analytical Model for Nonlinear, Single-Mode, Classical Rayleigh-Taylor Instability at Arbitrary Atwood Numbers. Physical Review Letters, 88, Article ID: 134502. http://dx.doi.org/10.1103/PhysRevLett.88.134502

[16] Mikaelian, K.O. (1989) Turbulent Mixing Generated by Rayleigh-Taylor and Richtmyer-Meshkov Instabilities. Physica D: Nonlinear Phenomena, 36, 343-357. http://dx.doi.org/10.1016/0167-2789(89)90089-4

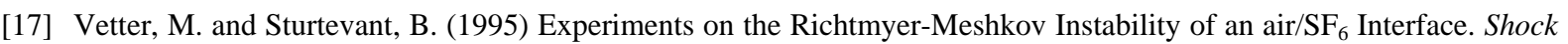
Waves, 4, 247-252. http://dx.doi.org/10.1007/BF01416035

[18] Leinov, E., Malamud, G., Elbaz, Y., Levin, L.A., Ben-Dor, G., Shvarts, D. and Sadot, O. (2009) Experimental and Numerical Investigation of the Richtmyer-Meshkov Instability under Re-Shock Conditions. Journal of Fluid Mechanics, 626, 449-475. http://dx.doi.org/10.1017/S0022112009005904

[19] Schilling, O. and Latini, M. (2010) High-Order WENO Simulation of Three-Dimensional Reshocked RichtmyerMeshkov Instability to Late Time: Dynamics, Dependence on Initial Conditions, and Comparisons to Experimental Data. Acta Mathematica Scientia, 30, 595-620. http://dx.doi.org/10.1016/S0252-9602(10)60064-1

[20] Mikaelian, K.O. (2011) Extended Model for Richtmyer-Meshkov Mix. Physica D: Nonlinear Phenomena, 240, 935-942. http://dx.doi.org/10.1016/j.physd.2011.01.008

[21] Mikaelian, K.O. (2015) Testing an Analytical Model for Richtmyer-Meshkov Turbulent Mixing Widths. Shock Waves, 25, 35-45. http://dx.doi.org/10.1007/s00193-014-0537-0

[22] Hill, D.J., Pantano, C. and Pullin, D.I. (2006) Large-Eddy Simulation and Multiscale Modelling of a RichtmyerMeshkov Instability with Reshock. Journal of Fluid Mechanics, 557, 29-61. http://dx.doi.org/10.1017/S0022112006009475

[23] Grinstein, F.F., Gowardhan, A.A. and Wachtor, A.J. (2011) Simulations of Richtmyer-Meshkov Instabilities in Planar Shock-Tube Experiments. Physics of Fluids, 23, Article ID: 034103.

[24] Vandenboomgaerde, M., Souffland, D., Mariani, C., Biamino, L., Jourdan, G. and Houas, L. (2014) An Experimental and Numerical Investigation of the Dependency on the Initial Conditions of the Richtmyer-Meshkov Instability. Physics of Fluids, 26, Article ID: 024109. http://dx.doi.org/10.1063/1.4865836

[25] Thornber, B., Drikakis, D., Youngs, D.L. and Williams, R.J.R. (2010) The Influence of Initial Conditions on Turbulent Mixing Due to Richtmyer-Meshkov Instability. Journal of Fluid Mechanics, 654, 99-139. http://dx.doi.org/10.1017/S0022112010000492

[26] Thornber, B., Drikakis, D., Youngs, D.L. and Williams, R.J.R. (2011) Growth of a Richtmyer-Meshkov Turbulent Layer after Reshock. Physics of Fluids, 23, Article ID: 095107. http://dx.doi.org/10.1063/1.3638616

[27] Luo, X.S., Si, T., Yang, J.M. and Zhai, Z.G. (2014) A Cylindrical Converging Shock Tube for Shock-Interface Studies. Review of Scientific Instruments, 85, Article ID: 015107. http://dx.doi.org/10.1063/1.4861357

[28] Si, T., Zhai, Z.G. and Luo, X.S. (2014) Experimental Study of Richtmyer-Meshkov Instability in a Cylindrical Converging Shock Tube. Laser and Particle Beams, 32, 343-351. http://dx.doi.org/10.1017/S0263034614000202

[29] Bai, J.S., Liu, J.H., Wang, T., Zou, L.Y., Li, P. and Tan, D.W. (2010) Investigation of the Richtmyer-Meshkov Instability with Double Perturbation Interface in Nonuniform Flows. Physical Review E, 81, Article ID: 056302.

[30] Bai, J.S., Wang, B., Wang, T. and Liu, K. (2012) Numerical Simulation of the Richtmyer-Meshkov Instability in Initially Nonuniform Flows and Mixing with Reshock. Physical Review E, 86, Article ID: 066319.

[31] Vreman, A.W. (2004) An Eddy-Viscosity Subgrid-Scale Model for Turbulent Shear Flow: Algebraic Theory and Applications. Physics of Fluids, 16, 3670-3681. http://dx.doi.org/10.1063/1.1785131

[32] Wang, T., Bai, J.S., Li, P. and Zhong, M. (2009) The Numerical Study of Shock-Induced Hydrodynamic Instability and Mixing. Chinese Physics B, 18, 1127-1135. http://dx.doi.org/10.1088/1674-1056/18/3/048

[33] Bai, J.S., Wang, T., Li, P., Zou, L.Y. and Liu, C.L. (2009) Numerical Simulation of the Hydrodynamic Instability Experiments and Flow Mixing. Science in China Series G, 52, 2027-2040. http://dx.doi.org/10.1007/s11433-009-0277-9

[34] Erez, L., Sadot, O., Oron, D., Erez, G., Levin, L.A., Shvarts, D. and Ben-Dor, G. (2000) Study of the Membrane Effect on Turbulent Mixing Measurements in Shock Tubes. Shock Waves, 10, 241-251. http://dx.doi.org/10.1007/s001930000053 